\title{
86810 - EVOLUÇÃO DO ESTADO NUTRICIONAL DE IDOSOS ATENDIDOS NA ATENÇÃO BÁSICA
}

\author{
Pôster - Gerontologia
}

\author{
Carolina Böettge Rosa / Rosa, CB / PUCRS; Dinara Hansen / Hansen, D / UNICRUZ; \\ Solange Billig Garces / Garces, SB / UNICRUZ; Ângela Vieira Brunelli / Brunelli, AV / UNICRUZ; \\ Carla Helena Augustin Schwanke / Schwanke, CHA / PUCRS
}

Introdução: $\mathrm{O}$ envelhecimento pode causar alterações no estado nutricional devido às diversas mudanças biopsicossociais inerentes a esse processo. Cabe a Atenção Básica monitorar essas mudanças, a fim de ordenar as prioridades de atendimento e promover intervenção preventiva nessa população. Objetivo: Analisar a evolução do estado nutricional de idosos atendidos na Atenção Básica num período de seguimento de três anos. Métodos: Foram entrevistados 375 idosos atendidos na Atenção Básica do município de Cruz Alta - RS, nos anos de 2012 e 2015. O estado nutricional foi avaliado através da Mini Avaliação Nutricional (MNA®). Para a análise da evolução do estado nutricional comparou-se as classificações categóricas da MNA® de dois momentos de avaliação (2012 e 2015), e as médias dos escores totais através de Teste t de Student, considerando-se $\mathrm{P}<0,05$. Resultados: A média de idade foi de 69,8 $\pm 7,25$ anos, variando de 60 a 92 anos. Ao comparar o estado nutricional dos dois momentos, observou-se que, a média do escore total da MNA® aumentou significativamente de 2012 para $2015(\mathrm{P}=0,021)$. Entretanto, após três anos da avaliação inicial, houve uma piora do estado nutricional em 10,7\% ( $\mathrm{N}=40)$ dos idosos. Conclusão: As deficiências nutricionais são consideradas condições sensíveis à Atenção Básica, ou seja, fazem parte de um conjunto de problemas de saúde para os quais a efetiva ação da Atenção Primária pode diminuir o risco de morbimortalidade. Os resultados sugerem a importância de se observar a evolução do estado nutricional em idosos da comunidade, como um indicador precoce para desfechos negativos nessa população.

Palavras-chave: Idoso; Estado nutricional; Atenção primária à saúde. 